\title{
ARTIGOS
}

Submetido 13.02.2019. Aprovado 12.06.2020

Avaliado pelo sistema double blind review, com isenção e independência. Editor Científico: Felipe Zambaldi

Versão original

DOI: http://dx.doi.org/10.1590/So034-759020210203

\section{ALAVANCANDO A EFICÁCIA DE SERVIÇOS INTENSIVOS EM CONHECIMENTO: O PAPEL DOS CLIENTES E DOS PRESTADORES DE SERVIÇO}

\author{
Leveraging the effectiveness of knowledge-intensive services: The roles of customers \\ and service providers \\ Optimizando la efectividad de los servicios intensivos en conocimiento: El papel de \\ los clientes y proveedores de servicios
}

\begin{abstract}
RESUMO
Esta pesquisa desenvolve um modelo teórico que relacionou as ações de participação dos clientes em serviços intensivos em conhecimento (SIC), as ações dos prestadores de serviço para estimular o envolvimento dos clientes e a eficácia dos serviços. Cinco hipóteses foram testadas por meio de equações estruturais, e os dados foram coletados por meio do levantamento de uma amostra de 106 usuários de serviços de fisioterapia, personal trainers, pilates e reeducação postural global. Os resultados mostram que não basta educar clientes; prestadores precisam ter empatia e criar um vínculo emocional com eles. Adicionalmente, a educação dos clientes e a empatia emocional influenciam de maneira diferenciada o fornecimento de feedback e até que ponto os clientes seguem as instruções. Este estudo contribui para a literatura, ao promover uma visão holística sobre os fatores que influenciam a participação do cliente e sua eficácia em SIC
\end{abstract}

PALAVRAS-CHAVE | Serviços intensivos em conhecimento, eficácia do serviço, participação do cliente, empatia emocional.

\section{ABSTRACT}

This research develops a theoretical model that related the actions of customers' participation in knowledge-intensive services (KIS), the actions of service providers to stimulate the involvement of customers and the effectiveness of services. Five hypotheses were tested using structural equations, and data were collected by surveying a sample of 106 users of physical therapy services, personal trainers, pilates and global postural reeducation. The results show that educating customers is not enough; providers need to empathize and create an emotional bond with them. Additionally, customer education and emotional empathy differently influence the provision of feedback and the extent to which customers follow instructions. This study contributes to the literature, by promoting a holistic view on the factors that influence the client's participation and its effectiveness in KIS.

KEYWORDS / Knowledge-intensive services, service effectiveness, customer participation, emotional empathy.

SIMONE SANTOS DA SILVA

\section{simonedt@gmail.com}

0o00-0002-2911-2407

JULIANA BONOMI SANTOS²

juliana.bonomi@fgv.br

oooo-0002-9582-7152

${ }^{1}$ Centro Universitário FEl, Programa de Pós graduação em Administração de Empresas, São Paulo, SP, Brasil

${ }^{2}$ Fundacao Getulio Vargas, Escola de Administração de Empresas de São Paulo, São Paulo, SP, Brasil

\section{RESUMEN}

Esta investigación desarrolla un modelo teórico que relaciona las acciones de participación del cliente en los servicios intensivos en conocimiento (SIC), las acciones de los proveedores de servicios para estimular la participación del cliente y la efectividad de los servicios. Se probaron cinco hipótesis utilizando ecuaciones estructurales, y los datos se recolectaron encuestando a una muestra de 106 usuarios de servicios de fisioterapia, entrenadores personales, pilates y reeducación postural global. Los resultados muestran que educar a los clientes no es suficiente; los proveedores deben sentir empatía y crear un vínculo emocional con ellos. Además, la educación del cliente y la empatía emocional influyen de manera diferente en la entrega de comentarios y en el grado en que los clientes siguen las instrucciones. Este estudio contribuye a la literatura, al promover una visión holística sobre los factores que influyen en la participación del cliente y su efectividad en la SIC.

PALABRAS CLAVE / Servicios intensivos en conocimiento, efectividad del servicio, participación del cliente, empatía emocional, encuesta. 


\section{INTRODUÇÃO}

Nos serviços intensivos em conhecimento (SIC), tais como consultoria financeira ou arquitetura, profissionais usam seus conhecimentos para desenvolver uma solução personalizada que atenda demandas ou resolva problemas dos clientes (Bettencourt, Ostrom, Brown, \& Roundtree, 2002; Santos \& Spring, 2015). 0 prestador precisa conhecer as necessidades do cliente e entender como elas mudam (Santos \& Spring, 2015). Por esse motivo, a participação do cliente é fundamental. Clientes participam informando suas necessidades e fornecendo feedback (Eisingerich \& Bell, 2006). Clientes também devem executar atividades e, por isso, é importante que sigam instruções passadas pelos provedores (Dellande, Gilly, \& Graham, 2004).

Prestadores devem educar o cliente, explicando conceitos e fornecendo informações para ajudá-los na compreensão do serviço (Eisingerich \& Bell, 2006). Clientes com maior entendimento tendem a envolver-se mais (Bell \& Eisingerich, 2007; Eisingerich \& Bell, 2008; Santos \& Spring, 2015). Prestadores com maior empatia emocional, isto é, maior capacidade de entender emoções, problemas e sentimentos do cliente (Lin, Yang, \& Huang, 2016), oferecem atendimento mais cortês (Wieseke, Geigenmuller, \& Kraus, 2012), estimulando o envolvimento do cliente devido à proximidade do relacionamento (Fang, Palmatier, \& Evans, 2008).

Estudos sobre ações de clientes e prestadores apontam que essas ações podem aumentar nível de qualidade do serviço (Dong, Sivakumar, Evans, \& Zou, 2015), lealdade (Auh, Bell, McLeod, \& Shih, 2007; Eisingerich \& Bell, 2006, 2008; Howcroft, Hamilton, \& Hewer, 2007), e satisfação (Dong et al., 2015; Eichentopf, Kleinaltenkamp, \& Stiphout, 2011).

No entanto, falta compreender se as ações dos clientes e prestadores contribuem para a eficácia dos SIC (Dellande et al., 2004). A maior parte dos estudos explora os ganhos ligados à satisfação do cliente e à lealdade (Dong et al., 2015; Eisingerich \& Bell, 2006, 2008). Com isso, não se sabe se as ações dos clientes e prestadores geram maior satisfação e lealdade devido aos resultados ou ao relacionamento.

Os estudos também adotam uma visão restrita sobre a contribuição de ambos para a eficácia do serviço. Alguns focam as implicações do envolvimento do cliente, sem considerar as ações dos provedores (Dellande et al., 2004; Eichentopf et al., 2011; Kelley \& Donnely, 1990; Larsson \& Bowen, 1989). Outros levam em consideração o papel dos provedores na educação do cliente, mas dedicam atenção apenas à forma como os clientes seguem instruções (Auh et al., 2007; Eichentopf et al., 2011;
Eisingerich \& Bell, 2006, 2008; Evans, Stan, \& Murray, 2008; Howcroft et al., 2007; Kelley \& Donnely, 1990; Larsson \& Bowen, 1989). Falta, portanto, uma visão mais ampla sobre a conexão entre as ações de ambos, e se estas contribuem para melhorar a eficácia.

O objetivo desta pesquisa foi analisar a relação entre as ações de clientes e prestadores e a eficácia dos SIC. Foi formulado um modelo teórico para capturar a influência da empatia emocional do prestador e de suas ações de educação na forma como o cliente fornece feedback e segue instruções, e a relação entre as ações de clientes e prestadores e a eficácia do serviço. Um levantamento de dados por meio de questionário (survey) com 106 clientes de personal trainers, fisioterapia, Pilates e Reeducação Postural Global (RPG) foi realizado para testar as hipóteses.

\section{FUNDAMENTAÇÃO TEÓRICA}

\section{Serviços intensivos em conhecimento (SIC)}

SIC- consistem na prestação de atividade profissional, utilizada para criar, acumular e disseminar conhecimentos, com o objetivo de promover 0 atendimento das necessidades dos clientes, de maneira personalizada, atendendo a demandas ou resolvendo problemas (Bettencourt et al., 2002; Santos \& Spring, 2015). A prestação desses serviços começa com a apresentação de uma necessidade do cliente ao prestador (Santos \& Spring, 2015). Este, com base em conhecimentos, faz o diagnóstico da situação e propõe uma solução. Caso o cliente concorde com a solução apresentada, o prestador a coloca em prática. Três características são relevantes para descrever esses serviços.

Primeiro, o provedor deve ter informação (Muller \& Zenker, 2001), experiência e habilidades de relacionamento para entender a necessidade do cliente, oferecer uma solução e promover o contato necessário com ele (Davenport, Jarvenpaa, \& Beers, 1996). Segundo, a solução é personalizada, não sendo passível revenda sem adaptação (Muller \& Zenker, 2001). Por último, o cliente deve fornecer informações sobre suas expectativas (Bettencourt, 1997; Eisingerich, Auh, \& Merlo, 2014). Durante a prestação do serviço, o cliente também precisa informar como suas demandas se alteram (Santos \& Spring, 2015), auxiliar o prestador de serviços a tomar decisões (Yim, Chan, \& Lam, 2012) e executar atividades que dependam dele para a implantação da solução (Bettencourt et al., 2002; Yim et al., 2012). Se o provedor não conseguir estimular essa participação, ele terá um desafio para entender as necessidades 
do cliente. No entanto, o conceito de participação do cliente é amplo, sem uma definição clara sobre seu escopo e fronteiras (Dong \& Sivakumar, 2017).

Diversos serviços podem ser enquadrados nessa classificação, como treinamento, orientação e consultoria, design e arquitetura, serviços médicos (Nordenflycht, 2010), assim como outros serviços de saúde, tais como fisioterapia, também podem ser considerados SICs. Esses serviços dependem do conhecimento do prestador para a criação de um diagnóstico e plano de tratamento customizado, além de serem ajustados de acordo com avanços no quadro dos pacientes.

\section{As ações de participação do cliente em SIC}

A participação do cliente em SIC pode ocorrer durante o serviço (Santos \& Spring, 2015). Clientes podem executar duas ações principais. A primeira é seguir as instruções passadas pelo profissional (Dellande et al., 2004), uma vez que o prestador possui conhecimentos para auxiliar o atingimento das metas. Eles também podem atuar fornecendo feedback sobre os resultados (Bettencourt, 1997), para que o profissional possa ajustar o serviço às suas necessidades; dessa forma, o cliente age fornecendo informações para que o prestador possa criar a solução adequada (Aarikka-Stenroos \& Jaakkola, 2012). Cabe ressaltar que clientes podem fazer elogios ou indicar pontos para melhoria, sendo que ambos os comentários podem ser usados pelo prestador para adaptar o serviço.

Assim, no caso de SIC, o cliente pode executar dois tipos de ações: oferecer sugestões construtivas (feedback) ao prestador (Eisingerich \& Bell, 2006) e seguir as instruções passadas (Dellande et al., 2004). Essas duas ações do cliente contribuem para a eficácia do SIC (Dellande et al., 2004). No entanto, nem sempre o cliente provê feedback ou segue corretamente as instruções. Para fazer isso, ele precisa ter consciência de seu papel (Bettencourt et al., 2002; LengnickHall, 1996; Mills \& Morris, 1986).

O prestador tem um papel importante na promoção da participação do cliente. Ele precisa educá-lo a participar, passando conhecimento de sua responsabilidade durante o serviço e das atividades que precisarão ser executadas, inclusive quando não estiverem juntos (Dellande et al., 2004). Assim, evitam-se problemas com fatos não esclarecidos e se reduz a possibilidade de críticas, já que as partes combinam entre si suas responsabilidades. A literatura mostra que, quando o prestador educa o cliente, este tende a participar mais e expor suas opiniões (Aarikka-Stenroos \& Jaakkola, 2012; Auh et al.,
2007; Eichentopf et al., 2011; Eisingerich \& Bell, 2006; Evans et al., 2008; Grönroos, 2008; Howcroft et al., 2007; Kelley \& Donnely, 1990; Santos \& Spring, 2015). Também relata a importância de 0 prestador possuir relação empática com seus clientes (Bowman, Heilman, \& Seetharaman, 2004).

No entanto, apesar de a literatura mostrar a contribuição das ações do cliente para a eficácia, e que ações de educação promovidas pelos prestadores estimulam a participação dos clientes, não se sabe o efeito das ações promovidas pelos prestadores para eficácia, e se ações de clientes e prestadores se complementam. A seguir, formulamos as hipóteses do estudo, para melhor explorar essa questão.

\section{FORMULAÇÃO DAS HIPÓTESES}

\section{A importância das ações do cliente para a eficácia de SIC}

O conceito de eficácia está relacionado ao atingimento de metas. Em SIC, a eficácia está associada à entrega de uma solução que atenda às necessidades do cliente, cuja participação afeta a eficácia (Tuli, Kohli, \& Bharadwaj, 2007). Assim, faz sentido avaliar os resultados pela ótica do cliente, ou seja, perguntando ao consumidor sobre suas expectativas e opiniões quanto aos resultados (Dellande et al., 2004), e sobre suas experiências com o serviço (Edvardsson, 2005; Klaus \& Maklan, 2012). A opinião dele é fundamental para entender se os resultados foram atingidos (Berry, Wall, \& Carbone, 2006).

Fornecer feedback e seguir as instruções podem contribuir para a eficácia do serviço (Dellande et al., 2004), por diferentes motivos. Ao fornecer feedback e informações sobre suas preferências, os clientes contribuem para o desenvolvimento de um plano alinhado a suas necessidades e dão aos prestadores a oportunidade de melhorar os serviços durante sua ocorrência (Santos \& Spring, 2015). Além disso, algumas atividades só podem ser executadas pelos clientes e, ao seguir as instruções, há maior chance de sucesso. Com isso, os clientes podem perceber falhas e solicitar ajuda para resolvê-las (Bettencourt et al., 2002). Ambas as ações do cliente em SIC parecem ser fundamentais para maior eficácia. Diante disso, formulamos duas hipóteses de pesquisa:

H1a: $\mathrm{O}$ ato de o cliente seguir instruções está positivamente associado à maior eficácia do serviço.

H1b: $\mathrm{O}$ ato de o cliente fornecer feedback está positivamente associado à maior eficácia do serviço. 


\section{As ações do prestador de serviço: o papel da educação do cliente}

A educação do cliente ocorre quando os prestadores explicam conceitos complexos para eles e fornecem informações apropriadas para ajudá-los na compreensão das ofertas (Eisingerich \& Bell, 2006). Prestadores podem apresentar casos de sucesso, em que mostram eventos que foram bem-sucedidos por causa da participação (Kelley \& Donnely, 1990). A educação do cliente visa aumentar o nível de conhecimento, para que ele sinta motivação e capacidade em participar (Eisingerich \& Bell, 2006).

As ações de educação do cliente geram maior interação com o prestador e maior clareza para seguir as instruções (Bowman et al., 2004), mesmo quando não estão juntos. Ao entender o serviço e o que é esperado dele, o cliente tem maior capacidade de fornecer informações e feedbacks relevantes. A literatura mostra que essas ações de educação influenciam o cliente a fornecer feedback e seguir as instruções passadas, principalmente porque aumentam seu nível de confiança em participar e expor sua opinião (Aarikka-Stenroos \& Jaakkola, 2012; Auh et al., 2007; Eichentopf et al., 2011; Eisingerich \& Bell, 2006; Evans et al., 2008; Grönroos, 2008; Howcroft et al., 2007; Kelley \& Donnely, 1990; Santos \& Spring, 2015). Diante disso, as seguintes hipóteses foram propostas:

Hza: A educação do cliente em SIC está positivamente associada ao ato de o cliente seguir instruções.

H2b: A educação do cliente em SIC está positivamente associada ao ato de o cliente fornecer feedback.

A educação do cliente faz com que fique mais claro para ele como suas ações podem contribuir para o atingimento dos objetivos e a eficácia do serviço. No entanto, uma melhora da eficácia só ocorrerá se o cliente fornecer mais feedback e seguir as instruções. Isto é, as ações do cliente medeiam a relação entre as ações de educação e a eficácia do serviço (Santos \& Spring, 2015). Não basta o cliente conhecer seu papel, ele precisa seguir as instruções passadas (Bowman et al., 2004) para atingir com mais facilidade os objetivos. Além disso, o prestador só poderá obter melhores informações para o serviço e oferecer uma solução mais adequada às necessidades do cliente se o cliente der feedback e atualizações sobre sua situação. Assim, as seguintes hipóteses foram sugeridas:

Hза: $\mathrm{O}$ ato de o cliente seguir instruções medeia a relação entre a educação do cliente e a eficácia do serviço;

$\mathrm{H} 3 \mathrm{~b}$ : $\mathrm{O}$ ato de o cliente fornecer feedback medeia a relação entre a educação do cliente e a eficácia do serviço.
As ações do prestador de serviço: o papel da empatia emocional

A literatura define empatia como cognitiva e emocional. A primeira é a capacidade de entender o estado mental dos outros, o que ajuda a prever os próximos passos e identificar se existe mentira na relação (Clark, Murfett, Rogers, \& Ang, 2016; Lee, 2014; Pizam, 2015; Tran et al., 2013; Wieseke et al., 2012). A empatia emocional é definida como o entendimento de emoções, de problemas e de sentimentos (Lin et al., 2016). Esta pesquisa utilizou a empatia emocional do prestador de serviço, percebida pelo cliente.

Quando o prestador possui empatia emocional com o cliente, ele consegue identificar e classificar os consumidores com base nas necessidades, emoções, sentimentos e sinais não verbais (Lin et al., 2016). Assim, ele pode fornecer orientações personalizadas e que façam mais sentido para os clientes. A forma como o prestador conduz o relacionamento é fundamental para garantir a efetividade do cliente em seguir as instruções (Bowman et al., 2004). Ao fornecer informações assertivas, aumentam as chances de o cliente seguir as instruções. Quando o provedor dá atenção às emoções dos clientes, ele consegue fazer com que estes sigam melhor as orientações (Dellande et al., 2004).

Muitas vezes, os consumidores desejam que a pessoa que os atende conecte-se com a sua situação, e não apenas escute seu problema, mesmo que isso não resulte na solução de sua necessidade (Gorry \& Westbrook, 2011). Como a empatia do prestador pode despertar sentimentos como carinho e generosidade (Lee, 2014), ela o leva a se comportar de maneira mais benevolente e atenciosa. Pode-se, então, criar uma conexão emocional entre as partes (Development, Hoffman, \& York, 2008). Dessa forma, foram propostas as seguintes hipóteses:

H4a: A empatia emocional do prestador está positivamente associada ao ato de o cliente seguir instruções.

H4b: A empatia emocional do prestador está positivamente associada ao ato de o cliente fornecer feedback.

Uma maior empatia emocional do prestador também o leva a se interessar mais pelas necessidades do cliente, gerando maior conhecimento e compreensão para alcançar os objetivos (Lin et al., 2016). Isso aumenta as chances de o prestador oferecer uma solução mais adequada e, com isso, mais eficaz. A empatia emocional do prestador, quando percebida pelo cliente, pode gerar uma conexão entre eles, e quanto maior a proximidade, maior será a probabilidade de gerar soluções confiáveis (Evans et al., 2008) e melhorar a eficácia (Eisingerich \& Bell, 2008). Assim, se poderia esperar que a empatia emocional do provedor contribuísse para a eficácia. No entanto, mesmo que se crie o 
vínculo, para alcançar os objetivos estabelecidos e encontrar a melhor solução para suas necessidades, o cliente deve realizar as ações sugeridas pelo prestador e fornecer informações para que ele adapte continuamente o serviço às suas necessidades. Dessa forma, apresentamos as duas últimas hipóteses deste estudo:

H5a: $\mathrm{O}$ ato de o cliente seguir instruções medeia a relação entre a empatia emocional do prestador de serviço e a eficácia do serviço.

H5b: $\mathrm{O}$ ato de o cliente fornecer feedback medeia a relação entre a empatia emocional do prestador de serviço e a eficácia do serviço.

A Figura 1 apresenta o modelo teórico que foi testado na pesquisa.

\section{Figura 1. Modelo teórico}

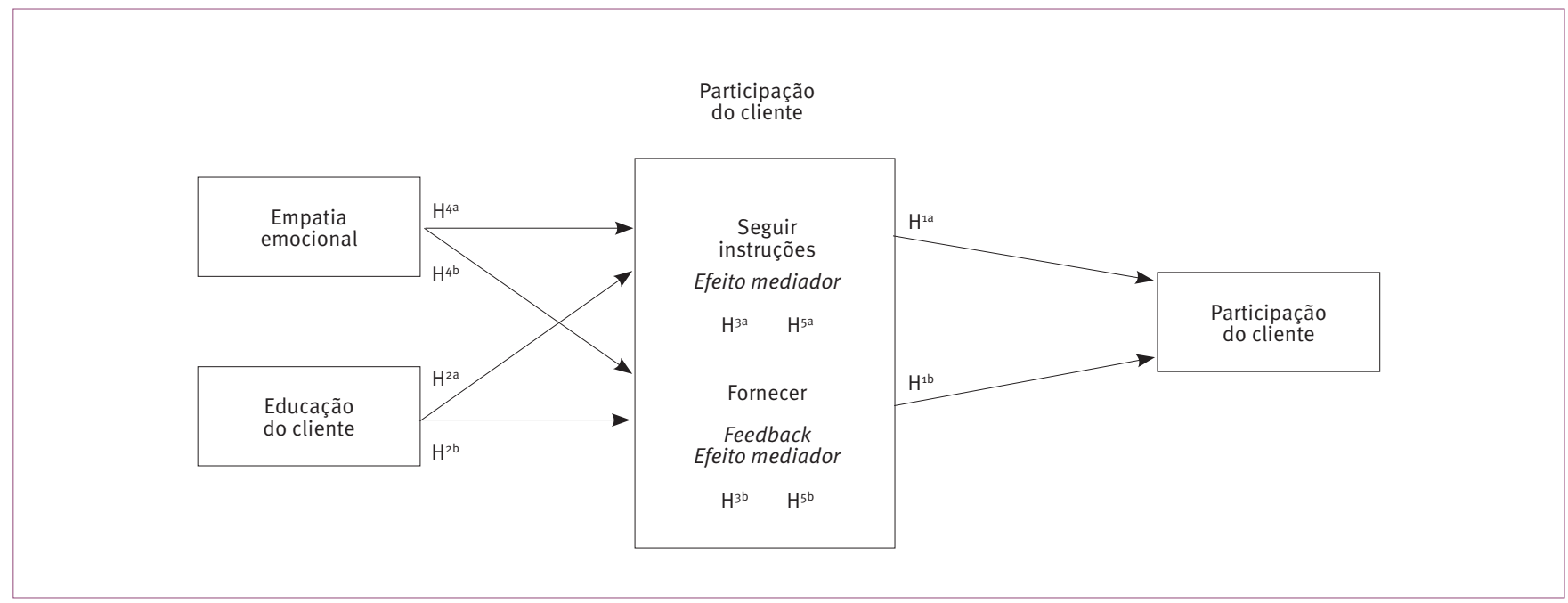

\section{METODOLOGIA}

Foi realizado um levantamento de dados por meio de questionário (survey) para testar as hipóteses formuladas sobre as relações entre fornecer feedback, seguir instruções, educar o cliente (Bell \& Eisingerich, 2007; Dellande et al., 2004; Eisingerich \& Bell, 2006), empatia emocional (Lin et al., 2016) e eficácia do serviço (Dellande et al., 2004).

\section{População e amostra}

A população desta pesquisa foi composta por usuários de SIC, especificamente no ramo de fisioterapia, personal trainers, RPG e pilates. Esses serviços podem ser considerados SIC, assim como outros serviços de diagnóstico prestados por médicos em clínicas e hospitais, pois o desenvolvimento depende do conhecimento específico do profissional que atende ao cliente. Além disso, nesses serviços, o programa de treinamento desenvolvido pelo profissional é personalizado e, para a sua criação, o cliente deve fornecer informações sobre suas expectativas, para que a solução seja alinhada à sua demanda. Por fim, durante a prestação do serviço, o cliente também precisa informar como suas demandas se alteram com o tempo para que o prestador ajuste o programa de treinamento.

Esses serviços também são relacionais, permitindo que os clientes forneçam feedback e que os prestadores tenham empatia pelos clientes. Tais semelhanças permitiram o uso desses diferentes serviços para o teste do modelo. Como todos os ramos pesquisados estão relacionados a um segmento específico do setor de saúde, espera-se uma certa homogeneidade entre o tipo de interação entre clientes e prestadores, feedbacks e ações de educação fornecidas, e a forma como clientes seguem instruções, restringindo a abrangência dos resultados a esse tipo de SIC.

$\mathrm{O}$ instrumento de coleta possuía três seções. A primeira fornecia orientações gerais sobre a forma de responder o questionário, identificar o tipo de serviço e a frequência de uso. A segunda seção continha uma escala para mensurar a participação do cliente (seguir instruções e fornecer feedback), as ações de educação do cliente, a empatia e a eficácia do serviço. As perguntas dessa seção foram apresentadas de maneira aleatória. O objetivo do questionário foi captar a percepção do cliente sobre cada construto. Assim, o modelo apresentado foi testado conforme a percepção do cliente. Ele foi aplicado por meio de 
ferramentas virtual e física. A terceira seção continha perguntas sobre o perfil dos respondentes.

O questionário final foi divulgado nas redes sociais, em canais como Linkedin, WhatsApp, e-mail e Facebook, por um período de dois meses. A importância da pesquisa foi descrita no instrumento de coleta de dados, e também foi realizada apresentação individual para as pessoas que responderam presencialmente. Também foi realizada coleta em academias e centros de reabilitação. A amostra utilizada, portanto, é não probabilística, assim, não permite a generalização dos resultados. Foram obtidas 94 respostas por meio eletrônico e 23 de questionários em papel, o que resultou em uma amostra final de 117 questionários. Testes t de Student para comparação de médias entre duas amostras indicaram não haver diferença significante ( $p$-valor > 0,05) entre os dados coletados por meio dos questionários eletrônicos e físicos. Foi feita uma triagem das respostas, e foram excluídos da análise 11 questionários, por apresentarem desvio-padrão entre as respostas menor do que 0,5. Assim, a amostra final foi composta por 106 questionários. A Tabela 1 apresenta as características da amostra.

A maior parte dos respondentes são usuários de personal trainers (59,2\%, vide Tabela 1$)$. Análises de variância para identificar diferenças entre as escalas, de acordo com o tipo de serviço, não indicaram diferenças significativas entre as variáveis ( $p>0,05)$. Esses testes permitiram que as análises fossem realizadas com a amostra total.

\section{Escalas adotadas}

O questionário foi desenvolvido a partir de escalas validadas em publicações anteriores. A escala de eficácia do serviço foi adaptada do trabalho de Dellande et al. (2004), em que os autores exploraram a opinião de clientes de serviços médicos em relação ao atingimento de metas, conceito semelhante ao adotado em nossa pesquisa. No entanto, aquele estudo avaliou questões ligadas ao estado dos clientes (por exemplo, disposição) e atingimento continuado de metas. Por esse motivo, optamos por trabalhar com os itens que avaliavam apenas o alcance de metas estabelecidas.

A participação do cliente, como definida neste estudo, busca capturar se ele segue as instruções passadas pelos provedores e fornece feedback. A escala relativa a seguir instruções foi obtida do trabalho de Dellande et al. (2004). As escalas sobre fornecimento de feedback por parte do cliente e de educação do cliente foram obtidas do trabalho de Eisingerich e Bell (2006). Por fim, a escala de empatia foi adaptada do estudo de Lin et al. (2016), que avaliaram a percepção de empatia na opinião do cliente. Dessa escala, optou-se por trabalhar apenas com os itens que mensuravam até que ponto o cliente percebia que o prestador estava atento às suas emoções, sentimentos e linguagens corporais (empatia emocional).

\section{Tabela 1. Características da amostra}

\begin{tabular}{|c|c|c|}
\hline $\begin{array}{l}\text { Características da } \\
\text { amostra }\end{array}$ & Classes & $\begin{array}{c}\text { Amostra }(n=106) \\
\text { Freq. } \%\end{array}$ \\
\hline \multirow{2}{*}{ Gênero } & Homem & 37,9 \\
\hline & Mulher & 62,1 \\
\hline \multirow{4}{*}{ Idade } & 31 ou menos & 29,2 \\
\hline & $31-45$ & 47,5 \\
\hline & $46-64$ & 21,4 \\
\hline & 65 ou mais & 1,9 \\
\hline \multirow{3}{*}{ Estado civil } & Solteiro & 43,7 \\
\hline & Casado & 47,6 \\
\hline & Outro & 8,7 \\
\hline \multirow{3}{*}{ Escolaridade } & $\begin{array}{l}\text { Ensino médio ou } \\
\text { técnico completo }\end{array}$ & 28,4 \\
\hline & $\begin{array}{l}\text { Ensino superior } \\
\text { completo }\end{array}$ & 31,4 \\
\hline & $\begin{array}{l}\text { Pós-graduação } \\
\text { completo }\end{array}$ & 40,2 \\
\hline \multirow{3}{*}{ Tipo de serviço } & Personal trainer & 59,2 \\
\hline & Fisioterapia e RPG & 19,4 \\
\hline & Pilates & 21,4 \\
\hline \multirow{2}{*}{$\begin{array}{l}\text { Tempo de } \\
\text { relacionamento }\end{array}$} & Menos de um ano & 56,3 \\
\hline & Mais de um ano & 43,7 \\
\hline \multirow{4}{*}{$\begin{array}{l}\text { Frequência de uso } \\
\text { do serviço }\end{array}$} & Todos dias & 12,6 \\
\hline & $\begin{array}{l}\text { Três vezes por } \\
\text { semana }\end{array}$ & 27.2 \\
\hline & $\begin{array}{l}\text { Duas vezes por } \\
\text { semana }\end{array}$ & 42.7 \\
\hline & $\begin{array}{c}\text { Uma vez por } \\
\text { semana ou menos }\end{array}$ & 17.5 \\
\hline
\end{tabular}

As escalas foram traduzidas para o português e adaptadas. Uma vez coletados os dados, realizaram-se análise das estatísticas descritivas e uma análise fatorial exploratória (AFE), com rotação varimax, para identificar a estrutura dimensional dos dados (Tabela 2). A AFE teve bom ajuste $(K M O=0,82)$, e o teste 
de Bartlett foi significante (p<0,01). A análise dos eigenvalues (acima de 1) sugeriu a existência de cinco fatores responsáveis por $75,4 \%$ da variância extraída. Uma variável da escala de educação do cliente foi eliminada, por apresentar carga de o,66 com seu fator, e 0,41 com o fator que continha as variáveis de empatia.

\section{Tabela 2. Escalas - Análise descritiva e cargas fatoriais da análise fatorial confirmatória}

\begin{tabular}{|c|c|c|c|c|}
\hline Construto & Escalas & Média & $\begin{array}{l}\text { Desvio- } \\
\text { Padrão }\end{array}$ & $\begin{array}{c}\text { Carga } \\
\text { Fatorial } \\
\text { AFC* }^{*}\end{array}$ \\
\hline Eficácia do serviço & Vou conseguir alcançar os objetivos que tracei inicialmente & 6 & 1,1 & ,84 \\
\hline \multirow[b]{3}{*}{ Seguir instruções } & $\begin{array}{l}\text { Continuo seguindo as orientações desse profissional mesmo quando não } \\
\text { estou com ele }\end{array}$ & 5,4 & 1,2 &, 77 \\
\hline & Para atingir meu objetivo, sigo todas as dicas desse profissional & 5,7 & 1,0 & ,74 \\
\hline & $\begin{array}{l}\text { Sigo as orientações desse profissional sobre o que é necessário fazer } \\
\text { antes e depois dos exercícios, mesmo quando ele não está por perto }\end{array}$ & 5,7 & 1,1 & ,74 \\
\hline \multirow{3}{*}{ Fornecer feedback } & $\begin{array}{l}\text { Faço sugestões construtivas para que esse profissional possa melhorar } \\
\text { seus serviços }\end{array}$ & 5,4 & 1,3 & 64 \\
\hline & $\begin{array}{l}\text { Quando eu tenho qualquer problema relacionado a esse profissional, } \\
\text { forneço feedback para que ele possa melhorar a qualidade do serviço }\end{array}$ & 5,6 & 1,2 & ,86 \\
\hline & $\begin{array}{l}\text { Quando esse profissional define exercícios de que eu gosto e que me dão } \\
\text { resultados, eu forneço feedback para que ele saiba disso }\end{array}$ & 6,1 & 1,1 & ,67 \\
\hline Educação do cliente & $\begin{array}{l}\text { Esse profissional explica conceitos técnicos dos exercícios recomendados, } \\
\text { e apresenta sempre os melhores caminhos }\end{array}$ & 5,7 & 1,3 & ,80 \\
\hline \multirow{3}{*}{ Empatia emocional } & $\begin{array}{l}\text { Percebo que esse profissional está atento às minhas emoções, } \\
\text { sentimentos e linguagens corporais }\end{array}$ & 5,3 & 1,3 & ,86 \\
\hline & $\begin{array}{l}\text { Esse profissional mostra-se preocupado com sentimentos que eu não } \\
\text { verbalizo }\end{array}$ & 5,1 & 1,4 & 98 \\
\hline & Esse profissional está atento aos meus sentimentos & 4,9 & 1,5 & ,68 \\
\hline
\end{tabular}

*significante ao nível o,01

\section{Análise de validade e confiabilidade}

Análise fatorial confirmatória foi realizada para avaliar a validade e confiabilidade das escalas, usando o software AMOS. Na Tabela 3, é possível verificar os índices de ajuste do modelo de mensuração. Os dados indicam o bom ajuste do modelo. 
Tabela 3. Índices de ajuste do modelo

\begin{tabular}{l|c|c}
\hline Índices & Modelo de mensuração & $\begin{array}{c}\text { Padrão de referência } \\
\text { (Kline, 2005) }\end{array}$ \\
\hline X2 - Qui-Quadrado & 165,4 & $\star \star$ \\
\hline GL - Graus de liberdade & 94 & ** \\
\hline TLI & $0,90,9$ & $>0,9$ \\
\hline CFI & 0,91 & $>0,9$ \\
\hline IFI & 0,92 & $<0,08$ \\
\hline RMSEA & 0,08 & $\leq 3,00$ \\
\hline X2 - Qui-Quadrado normado & 1,76 & $>$ \\
\hline
\end{tabular}

Realizou-se, então, a análise da validade convergente, discriminante e de confiabilidade. Todas as variáveis apresentaram carga fatorial acima de 0,5 e foram significantes ao nível de 0,01 (vide tabela 2). A variância média extraída (VME) para todos os construtos também ficou acima de 0,5. Assim, as escalas apresentaram validade convergente. Para avaliação da validade discriminante, foram calculadas as correlações quadráticas entre construtos, e essas foram comparadas com a variância média extraída (Tabela 4). É possível observar que todas as correlações entre construtos ao quadrado são inferiores às VME (destacadas em negrito na diagonal), indicando, assim, que existe validade discriminante entre todos os construtos.

Tabela 4. Confiabilidade composta (CC), variância média extraída (VME) e correlação entre construtos ao quadrado

\begin{tabular}{l|c|c|c|c|c|c}
\hline Construtos* & cC & $\begin{array}{c}\text { Empatia } \\
\text { emocional }\end{array}$ & $\begin{array}{c}\text { Educação do } \\
\text { cliente }\end{array}$ & $\begin{array}{c}\text { Fornecer } \\
\text { feedback }\end{array}$ & $\begin{array}{c}\text { Seguir } \\
\text { instruções }\end{array}$ & \\
\hline Empatia emocional & 0,84 & 0,65 & & & \\
\hline Educação do cliente & 0,85 & 0,25 & 0,66 & & \\
\hline Fornecer feedback & 0,77 & 0,27 & 0,26 & $\mathbf{0 , 5 4}$ & \\
\hline Seguir instruções & 0,84 & 0,03 & 0,24 & 0,11 & $\mathbf{0 , 5 6}$ & 0,28 \\
\hline Eficácia do serviço & 0,86 & 0,09 & 0,13 & 0,10 & 0,67 \\
\hline
\end{tabular}

*VME na diagonal

Por fim, calculou-se a confiabilidade composta, que foi superior a 0,7 em todos os casos (Tabela 4). Em conjunto, as análises apresentadas indicaram a validade e confiabilidade das escalas. Devido ao tamanho da amostra, optou-se por trabalhar com as escalas calculadas para cada construto. Para prosseguir com as análises, solicitou-se ao software AMOS que calculasse o valor dos fatores (escalas) e os salvasse na base de dados.

\section{APRESENTAÇÃO DOS RESULTADOS}

O método path analysis foi escolhido para testar as hipóteses. Antes de realizar essa análise, foi feita uma avaliação do impacto das variáveis de controle. Para isso, calculou-se uma regressão linear na qual a variável eficiência do serviço era a variável dependente, e as variáveis de controle eram as variáveis independentes. As variáveis de controle utilizadas foram: gênero, idade, estado civil, escolaridade, tipo de serviço, frequência de uso do serviço e tempo de relacionamento. Variáveis dummy foram criadas para representar as classes das variáveis de controle (Tabela 1). A análise de regressão apresentou $R^{2}$ de 5,3\%, e a análise de variância não foi significativa $(p=0,91)$, indicando a falta de relação entre, ao menos, uma variável de controle e a eficiência do serviço.

Como nenhuma variável de controle mostrou-se significante, essas não foram inseridas no path analysis. 0 modelo apresentou bom ajuste, uma vez que todos os indicadores ficaram acima dos padrões de referência (Tabela 5 ). $O \mathrm{R}^{2} \mathrm{da}$ regressão em relação à eficácia do serviço foi de 0,51. Os $R^{2}$ para as variáveis "seguir instruções" e "fornecer feedback" foram 0,51 
e 0,30 , respectivamente. Os valores dos quadrados indicaram que as variáveis independentes do modelo explicavam uma parcela relevante da variabilidade das variáveis em questão.

Tabela 5. Índice de ajuste do modelo para path analysis

\begin{tabular}{l|c|c}
\hline Índices & Ajuste do modelo & $\begin{array}{c}\text { Padrão de referência } \\
\text { (Kline, 2005) }\end{array}$ \\
\hline X2 - Qui-Quadrado & 2,68 & $\star *$ \\
\hline GL - Graus de liberdade & 1 & ** \\
\hline TLI & 0,92 & $>0,9$ \\
\hline CFI & 0,99 & $>0,9$ \\
\hline IFI & 0,99 & $>0,9$ \\
\hline X2 - Qui-Quadrado normado & 2,68 & $\leq 3,00$ \\
\hline
\end{tabular}

Após a análise da adequação do modelo, foram avaliados os coeficientes das regressões entre construtos, para testar as hipóteses propostas (Figura 2). A relação entre seguir instruções e a eficácia do serviço mostrou-se significante, mas o mesmo não ocorreu para a relação entre oferecer feedback e a eficácia do serviço. Assim, há evidências estatísticas que dão suporte para $H^{1 a}$, mas não para $\mathrm{H}^{1 \mathrm{~b}}$.

Figura 2. Teste das hipóteses

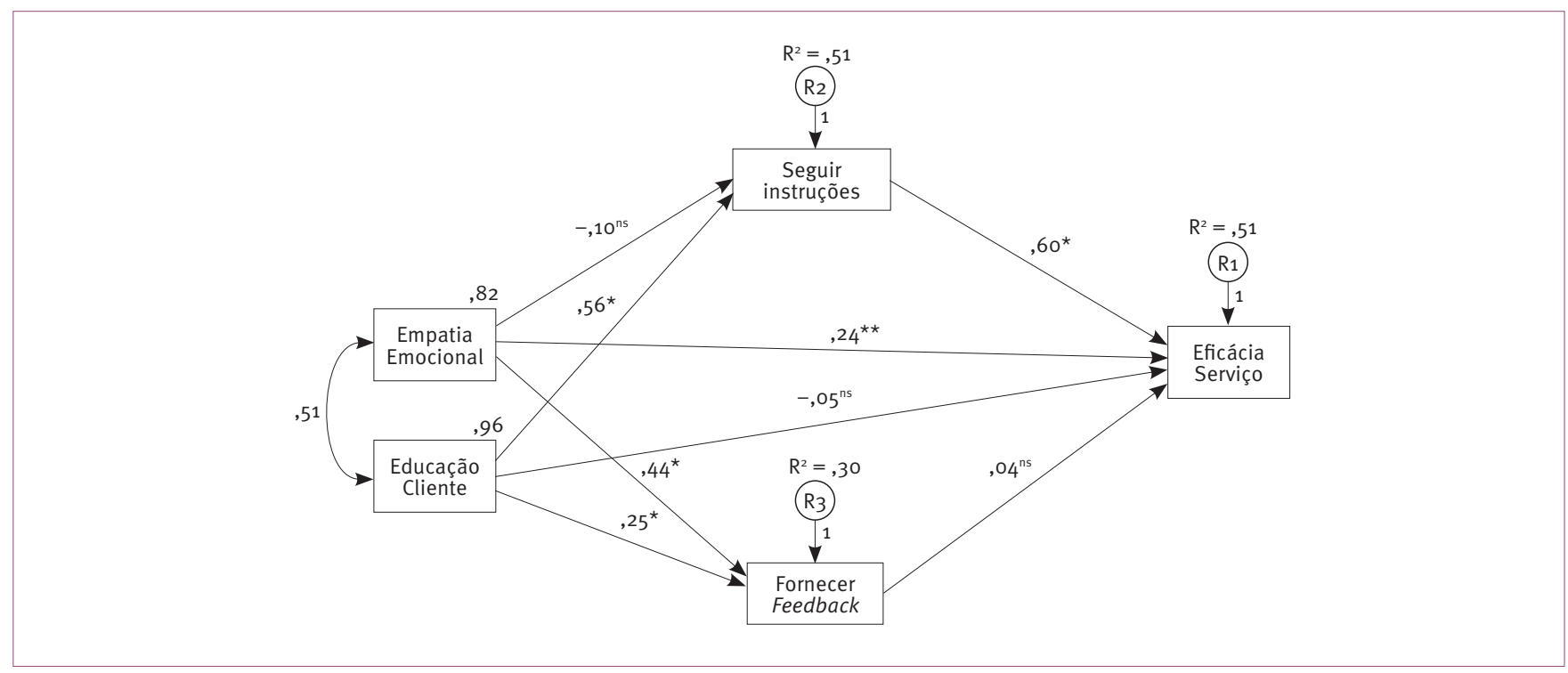

${ }^{*} \mathrm{p}<, 01 ;{ }^{* *} \mathrm{p}<, 05 ;$ ns=não significante

Os resultados também indicam que as ações de educação do cliente estão positivamente associadas ao fornecimento de feedback e ao fato de o cliente seguir as instruções do prestador, comprovando $\mathrm{H}^{2 \mathrm{a}}$ e $\mathrm{H}^{2 b}$.

Para testar a hipótese de mediação de seguir instruções e fornecer feedback, na relação entre educação e eficácia do serviço $\left(\mathrm{H}^{3 \mathrm{a}}\right.$ e $\left.\mathrm{H}^{3 \mathrm{~b}}\right)$, estimou-se o efeito combinado das relações entre educação do cliente e as variáveis mediadoras (seguir instruções e fornecer feedback) (A) e das variáveis mediadoras e a eficácia do serviço (B). Para realizar as estimações, utilizou-se bootstrapping com geração de 2 mil amostras, com o auxílio do software AMOS. No caso da variável mediadora "seguir instruções", o efeito combinado (AxB) foi de 0,33 e significante $(p<0,01)$. Já no caso da variável mediadora "fornecer feedback", o efeito combinado das relações entre educação do cliente e fornecer feedback (A) e entre fornecer feedback e a eficácia do 
serviço (B) foi de $0,01(p=0,76)$. Assim, $\mathrm{H}^{3 a}$ foi confirmada, mas não foi possível confirmar $\mathrm{H}^{3 \mathrm{~b}}$. Estimou-se também o efeito direto entre ações de educação e a eficácia do serviço (C), que não foi significante $(p=0,62)$. Esse achado indica uma mediação total da variável "seguir instruções" na relação entre a educação do cliente e a eficácia do serviço.

A empatia emocional mostrou-se positivamente associada ao fornecimento de feedback (p<0,01), mas não se relacionou com o fato de o cliente seguir instruções, confirmando apenas $\mathrm{H}^{4 a}$, mas não $\mathrm{H}^{4 \mathrm{~b}}$. Por fim, a hipótese de mediação de seguir instruções e fornecer feedback, na relação entre empatia emocional e a eficácia do serviço $\left(\mathrm{H}^{5 a}\right.$ e $\left.\mathrm{H}^{5 b}\right)$, foi testada da mesma forma que se testaram $\mathrm{H}^{3 a}$ e $\mathrm{H}^{3 b}$. $\mathrm{O}$ efeito combinado das relações entre empatia e seguir instruções $(A)$ e entre seguir instruções e a eficácia do serviço (B) foi de -0,06 e não significante $(p=0,16)$. 0 efeito combinado das relações entre empatia e fornecimento de feedback (A) e entre fornecimento de feedback e a eficácia do serviço (B) foi de 0,017 e não foi significante $(p=0,78)$. Assim, $H^{5 a} e$ $\mathrm{H}^{5 b}$ não foram confirmadas. No entanto, identificou-se uma relação direta entre empatia emocional e a eficácia do serviço, que se mostrou significante $(b=0,24 ; p<0,05)$. A Tabela 6 apresenta 0 resumo das hipóteses.

\section{Tabela 6. Resumo das hipóteses}

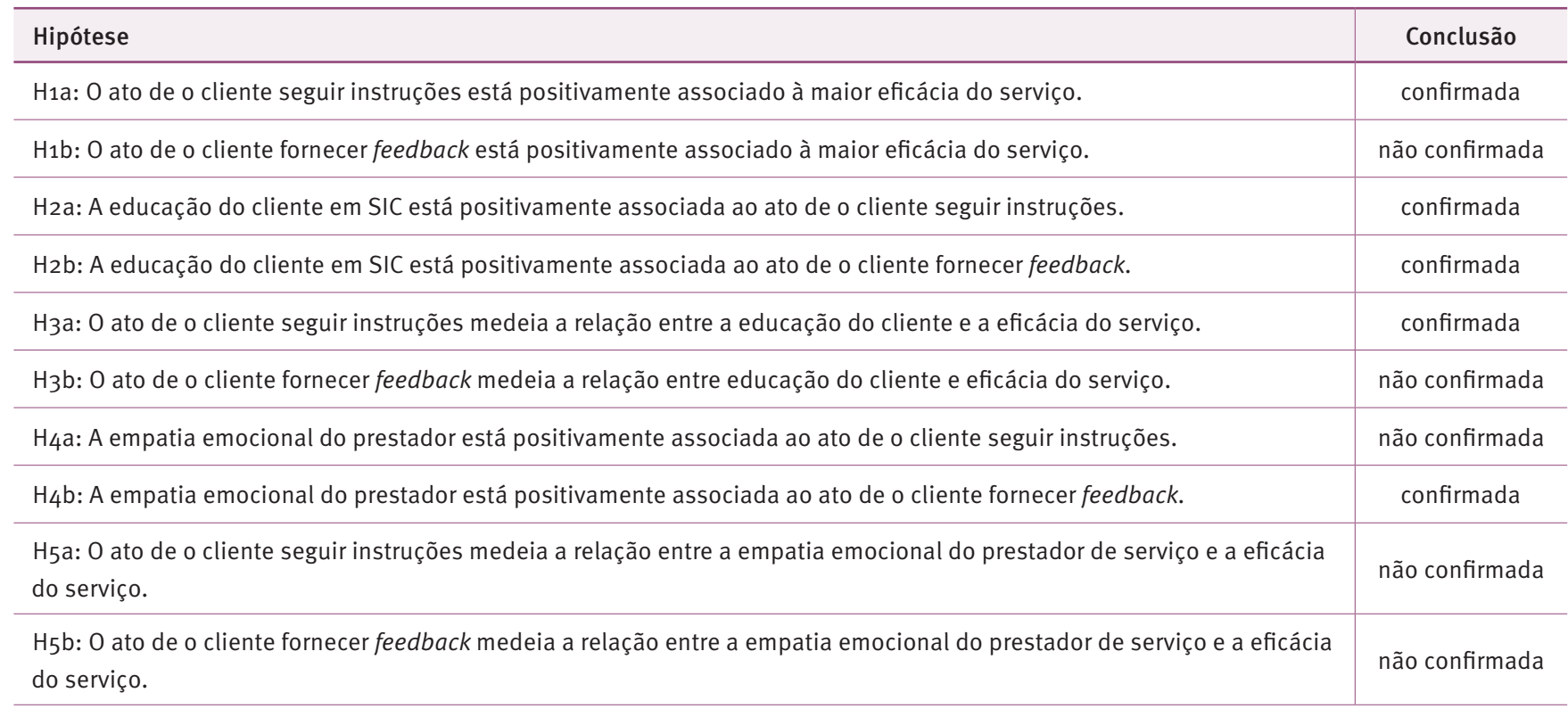

\section{DISCUSSÃO}

A literatura já mostrou que a participação do cliente está ligada à satisfação, confiança e lealdade (Eisingerich \& Bell, 2006; Fang et al., 2008; Jamal, 2009; Reichheld \& Sasser, 1990; Sabharwal, Soch, \& Kaur, 2010; Zeithaml, Berry, \& Parasuraman, 1996). Nosso estudo mostra que o envolvimento dos clientes também gera maior sensação de eficácia nos clientes de SIC. Maior eficácia está relacionada apenas ao ato de os clientes seguirem as instruções passadas, em linha com o estudo de Dellande et al. (2004). Ou seja, no caso dos serviços estudados, quanto mais os indivíduos seguirem as orientações, tais como manter a postura correta quando sozinhos e manter uma alimentação balanceada, maior é a chance de alcançarem seus objetivos de perda de peso ou melhoria da qualidade de vida. Por outro lado, o fornecimento de feedback não contribui para o alcance desses objetivos. A literatura, no entanto, aponta que o feedback é importante para maior lealdade do cliente (Eisingerich \& Bell, 2006). Portanto, é possível que ele seja importante para melhorar a relação entre as partes, mas não é suficiente para levar o cliente a alcançar eficácia.

Este estudo contribui para a literatura ao oferecer uma visão integrada sobre a participação dos clientes em SIC e o papel dos provedores. Até então, os estudos focavam apenas as ações dos clientes (Dellande et al., 2004; Eichentopf et al., 2011; Kelley \& Donnely, 1990; Larsson \& Bowen, 1989) ou abordavam somente uma forma de atuação dos clientes (Auh et al., 2007; Eichentopf et al., 2011; Eisingerich \& Bell, 2006, 2008; Evans et al., 2008; Howcroft et al., 2007; Kelley \& Donnely, 1990; Larsson \& Bowen, 1989). Neste estudo, identificou-se que o ato de fornecer feedback e seguir instruções são antecedentes, e possuem implicações diferenciadas. Os resultados indicam que o fornecimento de 
feedback está associado à empatia emocional do prestador e às ações de educação do cliente, mas não à maior eficácia. Já o fato de os clientes seguirem instruções está relacionado com melhor eficácia, mas parece derivar apenas das ações de educação do prestador, e não de sua empatia. No caso dos serviços estudados, pode-se dizer, então, que, ao serem empáticos, personal trainers, professores de Pilates e de outras modalidades semelhantes têm maior chance de fazer com que seus clientes forneçam sugestões para a melhoria do serviço, mesmo que isso não se traduza em melhores resultados. Por outro lado, eles só conseguirão fazer com que seus clientes alcancem suas metas pessoais se fornecerem orientação detalhada de como eles devem agir fora das aulas.

Por fim, este estudo avalia empiricamente o papel da empatia do prestador, e aponta que ela está associada a mais fornecimento de feedback e ao atingimento de metas. Assim, se o prestador estiver atento às emoções e sentimentos dos clientes, ele os motivará a dizer se o serviço está bom ou ruim e perceber os resultados mais positivamente. Com isso, este estudo contribui para a literatura ao destacar a importância de aspectos relacionais na prestação de SIC e traz para a discussão a necessidade de se considerarem características intrínsecas do prestador. A educação do cliente já foi tratada em diversos contextos na literatura (Auh et al., 2007; Eichentopf et al., 2011; Eisingerich \& Bell, 2006, 2008; Evans et al., 2008; Howcroft et al., 2007; Kelley \& Donnely, 1990; Larsson \& Bowen, 1989), porém este trabalho mostra a importância da empatia do provedor, e novos estudos devem continuar pesquisando outras ações dos prestadores que sejam relevantes.

A presente pesquisa também traz implicações gerenciais para os profissionais que atuam em SIC. Eles devem reservar tempo para explicar ao cliente como agir, mesmo quando não estejam juntos, e ressaltar a importância das atividades que são feitas na ausência do prestador para a obtenção de resultados. No survey utilizado, as pessoas que sabiam da importância de certos movimentos continuavam a fazê-los mesmo na ausência de seu prestador. Atualmente, com as redes sociais, o contato entre prestadores e clientes é mais fácil e rápido. Assim, prestadores podem desenvolver estratégias de contato ativo, na qual enviam mensagens, com lembretes e sugestões de atividades.

Esse tipo de contato também favorece o relacionamento, fazendo com que fiquem mais próximos e que o cliente perceba a empatia do prestador em relação a suas necessidades. Quando o cliente sente confiança e desenvolve laços sociais com prestador, aumenta sua disposição em participar e expor sua opinião, fornecendo feedback (Eisingerich \& Bell, 2006). Esse relacionamento pode também aumentar a tolerância do cliente ao erro.
Considerando os achados deste estudo em relação à empatia, sugere-se que o prestador adote uma postura que demonstre sua empatia. Ao perceber que existe abertura por parte do cliente, é importante perguntar sobre questões pessoais, solidarizar-se com histórias e acontecimentos, avaliar o humor do cliente e adequar a prestação do serviço à situação. Sabe-se que indivíduos possuem diferentes níveis de empatia (Lee, 2014), mas a empatia pode ser desenvolvida por meio de treinamentos sobre a forma de interagir com clientes, conforme as percepções do prestador (Lee, 2014). Assim, prestadores com dificuldade em se relacionar devem investir em treinamentos.

\section{CONCLUSÃO}

Os resultados mostram que não basta apenas educar os clientes. Prestadores precisam ser empáticos e criar um vínculo emocional com eles. Prestadores empáticos e que educam seus clientes estimulam mais a participação e, assim, obtêm melhores resultados. Esta pesquisa mostra que há diferenças na forma como os clientes participam, e um melhor entendimento sobre a participação do cliente em SIC requer uma avaliação das diferentes dimensões da participação. Esses achados são relevantes e abrem novas rotas de pesquisa, além de direcionar o comportamento dos prestadores para que possam obter melhores resultados.

As pessoas que responderam ao questionário são usuárias de quatro tipos de serviços (fisioterapia, personal trainers, Pilates e RPG), todos ligados à saúde, e a maior parte dos respondentes utiliza o serviço de personal trainers. Assim, os resultados deste trabalho são limitados a esse contexto. Além disso, o estudo baseia-se em uma amostra não probabilística, o que não permite a generalização dos resultados. Embora as contribuições sejam relevantes, estudos futuros devem pesquisar outros serviços, como consultoria financeira, para identificar se os resultados encontrados podem ser aplicados.

Futuros estudos devem explorar, em maior detalhe, outras variáveis ligadas ao relacionamento entre as partes e como elas influenciam a participação. Dado que a empatia emocional do prestador se mostrou relevante, e uma das premissas adotadas é que isso ocorreu por ela fortalecer o relacionamento, faz sentido estudar o relacionamento em maior profundidade. Existem outras estratégias para estimular a participação, tais como socialização do cliente e gerenciamento de problemas. Na socialização, o provedor pode levar o cliente a observar casos de outros clientes, parecidos com o seu, ou criar eventos para interagir com ele fora do horário de prestação do serviço, motivando sua participação 
(Evans et al., 2008). Já no gerenciamento de problemas, o provedor resolve uma determinada situação, e o cliente fica com a sensação de que suas necessidades são levadas em consideração. Essas ações reforçam o relacionamento, fazendo com que o cliente participe mais (Eisingerich \& Bell, 2006; Santos \& Spring, 2015). Novos estudos devem explorar a relação entre a empatia do prestador e essas outras estratégias, levando em consideração a estratégia mais debatida na literatura - educar o cliente. Futuras pesquisas devem explorar fatores que impedem a participação efetiva do cliente em direção aos resultados esperados. Eficácia, aqui, foi tratada como a percepção do resultado por parte do cliente. Em futuros estudos, poderia ser investigada a relação entre a eficácia real e as ações de clientes e prestadores. Isso demonstraria se essas ações têm efeito real ou apenas influenciam a percepção de resultados pelo cliente.

\section{REFERÊNCIAS}

Aarikka-Stenroos, L., \& Jaakkola, E. (2012). Industrial marketing management value co-creation in knowledge intensive business services: A dyadic perspective on the joint problem solving process, 41, 15-26. doi: 10.1016/j.indmarman.2011.11.008

Auh, S., Bell, S. J., McLeod, C. S., \& Shih, E. (2007). Co-production and customer loyalty in financial services. Journal of Retailing, 83(3), 359370. doi:10.1016/j.jretai.2007.03.001

Bell, S. J., \& Eisingerich, A. B. (2007). The paradox of customer education. European Journal of Marketing, 41(5/6), 466-486. doi:10.1108/03090560710737561

Berry, L. L., Wall, E. A., \& Carbone, L. P. (2006). Service clues and customer assessment of the service experience: Lessons from marketing. Academy of Management Perspectives, 20(2), 43-57. doi:10.5465/AMP.2006.20591004

Bettencourt, L., Ostrom, A., Brown, S., \& Roundtree, R. (2002). Client co-production in knowledge-intensive business services. Operations Management: A Strategic Approach, 44(4), 273.

Bettencourt, L. A. (1997). Customer voluntary performance: Customers as partners in service delivery. Journal of Retailing, 73(3), 383-406. doi: 10.1016/Soo22-4359(97)90024-5

Bowman, D., Heilman, C. M., \& Seetharaman, P. B. (2004). Determinants of product-use compliance behavior. Journal of Marketing Research, 41(3), 324-338. doi:10.1509/jmkr.41.3.324.35987

Clark, C. M., Murfett, U. M., Rogers, P. S., \& Ang, S. (2016). Is empathy effective for customer service? Evidence from call center interactions. Journal of Business and Technical Communication, 27(2), 123-153. doi: 10.1177/1050651912468887

Davenport, T. H., Jarvenpaa, S. L., \& Beers, M. C. (1996). Improving knowledge work processes. Sloan Management Review, 37(4), 52-65.

Dellande, S., Gilly, M. C., \& Graham, J. L. (2004). Gaining compliance and losing weight: The role of the service provider in health care services. Journal of Marketing, 68(3), 78-91. doi: 10.1509/jmkg.68.3.78.34764
Development, M., Hoffman, B. M. L., \& York, N. (2008). Book reviews. Journal of Travel \& Tourism Marketing, 24(4), 321-325. doi: $10.1080 / 10548400802156836$

Dong, B., \& Sivakumar, K. (2017). Customer participation in services: Domain, scope, and boundaries. Journal of the Academy of Marketing Science, 45(6), 944-965.

Dong, B., Sivakumar, K., Evans, K. R., \& Zou, S. (2015). Effect of customer participation on service outcomes: The moderating role of participation readiness. Journal of Service Research, 18(2), 160-176. doi: $10.1177 / 1094670514551727$

Edvardsson, B. (2005). Service quality: Beyond cognitive assessment. Managing Service Quality: An International Journal, 15(2), 127-131. doi: $10.1108 / 09604520510585316$

Eichentopf, T., Kleinaltenkamp, M., \& Stiphout, J. Van. (2011). Modelling customer process activities in interactive value creation. Journal of Service Management, 22(5), 650-663. doi: 10.1108/09564231111174997

Eisingerich, A., \& Bell, S. (2008). Customer education increases trust. MIT Sloan Management Review, 50(1), 10.

Eisingerich, A. B., Auh, S., \& Merlo, O. (2014). Acta non verba? The role of customer participation and word of mouth in the relationship between service firms' customer satisfaction and sales performance. Journal of Service Research, 17(1), 40-53. doi: 10.1177/1094670513490836

Eisingerich, A. B., \& Bell, S. J. (2006). Relationship marketing in the financial services industry: The importance of customer education, participation and problem management for customer loyalty. Journal of Financial Services Marketing, 10(4), 86-97. doi:10.1057/palgrave. fsm. 4760022

Evans, K. R., Stan, S., \& Murray, L. (2008). The customer socialization paradox: The mixed effects of communicating customer role expectations. Journal of Services Marketing, 22(3), 213-223. doi:10.1108/08876040810871174

Fang, E., Palmatier, R. W., \& Evans, K. R. (2008). Influence of customer participation on creating and sharing of new product value. Journal of the Academy of Marketing Science, 36(3), 322-336. doi:10.1007/ s11747-007-0082-9

Gorry, G. A., \& Westbrook, R. A. (2011). Once more, with feeling: Empathy and technology in customer care. Business Horizons, 54(2), 125-134. http://doi.org/10.1016/j.bushor.2010.10.003

Grönroos, C. (2008). Service logic revisited: Who creates value? And who co-creates? European Business Review, 20(4), 298-314. doi: 10.1108/09555340810886585

Howcroft, B., Hamilton, R., \& Hewer, P. (2007, January). Customer involvement and interaction in retail banking: An examination of risk and confidence in the purchase of financial products. Journal of Services Marketing, 21, 481-491. doi:10.1108/08876040710824843

Jamal, A. (2009). Investigating the effects of service quality dimensions and expertise on loyalty. European Journal of Marketing, 43(3/4), 398-420. doi:10.1108/03090560910935497

Kelley, S. W., \& Donnely, J. H. (1990). Customer participation in service specification and delivery. Journal of Retailing, 66(3), 315-335.

Klaus, P., \& Maklan, S. (2012). EXQ: A multiple item scale for assessing service experience. Journal of Service Management, 23(1), 5-33. doi: $10.1108 / 09564231211208952$ 
Larsson, R., \& Bowen, D. E. (1989). Organization and customer: Managing design and coordination of services. Academy of Management Review, 14(2), 213-233. doi: 10.5465/AMR.1989.4282099

Lee, E. J. (2014). Empathy can increase customer equity related to pro-social brands. Journal of Business Research, 69(9), 3748-3754. http://doi.org/10.1016/j.jbusres.2015.05.018

LengnickHall, C. A. (1996). Customer contribution to quality: A different view of the customer-oriented firm. Academy of Management Review, 21(3), 791-824. doi: 10.2307/259002

Lin, C.-P., Yang, Z.-T., \& Huang, H.-T. (2016). Evaluating team performance and the mediating role of customer knowledge development: An absorptive capacity framework. Journal of Engineering and Technology Management, 1-19. doi: 10.1016/j. jengtecman.2016.10.001

Mills, P. K., \& Morris, J. H. (1986). Clients as “ partial ” employees of service organizations: Role development in client participation. Academy of Management Review, 11(4), 726-735.

Muller, E., \& Zenker, A. (2001). Business services as actors of knowledge transformation: The role of KIBS in regional and national innovation systems. Research Policy, 30(9), 1501-1516. doi: 10.1016/Soo487333(01)00164-0

Nordenflycht, A. Von. (2010). What is a professional service firm? Toward a theory and taxonomy of knowledge-intensive firms. Academy of Management Review, 35(1), 155-174.

Pizam, A. (2015). Is empathy essential for high-quality customer service? International Journal of Hospitality Management, 49, 149150. doi: 10.1016/j.ijhm.2015.08.001
Reichheld, F. F., \& Sasser, W. E. (1990). Zero defections: Quality comes to services. Harvard Business Review, 68(105). doi: $10.1108 / 17506200710779521$

Sabharwal, N., Soch, H., \& Kaur, H. (2010). Are we satisfied with incompetent services? A scale development approach for service recovery. Journal of Services Research, 10(1), 125-142.

Santos, J. B., \& Spring, M. (2015). Are knowledge intensive business services really co-produced? Overcoming lack of customer participation in KIBS. Industrial Marketing Management, 50, 85-96. doi: 10.1016/j.indmarman.2015.04.005

Tran, U. S., Laireiter, A.-R., Schmitt, D. P., Neuner, C., Leibetseder, M., Szente-Voracek, S. L., \& Voracek, M. (2013). Factorial structure and convergent and discriminant validity of the e (empathy) Scale. Psychological Reports, 113(2), 441-463. doi: 10.2466/03.02. PRo.113X20z9

Tuli, K. R., Kohli, A. K., \& Bharadwaj, S. G. (2007). Rethinking customer solutions: From product bundles to relational processes. Journal of Marketing, 71(3), 1-17. doi: 10.1509/jmkg.71.3.1

Wieseke, J., Geigenmuller, A., \& Kraus, F. (2012). On the role of empathy in customer-employee interactions. Journal of Service Research, 15(3), 316-331. doi: 10.1177/1094670512439743

Yim, C. K. (Bennett), Chan, K. W., \& Lam, S. S. (2012). Do customers and employees enjoy service participation? Synergistic effects of self- and other-efficacy. Journal of Marketing, 76(6), 121-140. doi: 10.1509/jm.11.0205

Zeithaml, V. A., Berry, L., \& Parasuraman, A. (1996). The behavioral consequences of service quality. Journal of Marketing, 6o(2), 31-46. doi: $10.2307 / 1251929$

\section{CONTRIBUIÇÃO DAS AUTORAS}

As autoras declaram que a análise de dados, redação e revisão final foi realizada em conjunto pelas duas autoras. $A$ conceitualização, abordagem teórica-metodológica, revisão teórica e coleta de dados foi realizada em maior parte pela primeira autora. 